\title{
Effect of dispersion method in the production of Al-CNTs nanocomposites
}

\author{
Sónia Simões ${ }^{1}$, Filomena Viana ${ }^{1}$, Marcos A. L. Reis ${ }^{2}$ and Manuel F.Vieira ${ }^{1}$ \\ ${ }^{1 .}$ CEMUC, Department of Metallurgical and Materials Engineering, University of Porto, R. Dr. Roberto \\ Frias, 4200-465 Porto, Portugal. \\ ${ }^{2}$ Faculdade de Ciências Exatas e Tecnologia, Universidade Federal do Pará, Abaetetuba, PA 68440-000 \\ Brazil.
}

Nanocomposites reinforced with carbon nanotubes (CNTs) have attracted growing attention from the research community. The biggest challenge for the production of these composites is the development of processes that promote a dense and uniform dispersion of CNTs, undamaged and well bonded to the matrices, to accomplish its full reinforcement potential and achieve an effective load transfer. The production process of the nanocomposites has a great influence on the dispersion of the reinforcement [1-3].

This research is focused on the study of CNTs dispersion in Al-CNTs nanocomposites. The nanocomposites were produced by conventional powder metallurgy route. CNTs used in this work (from composites Fibermax) are mostly multi-walled CNTs (MWCNTs) and powders of aluminum (from Goodfellow) exhibit a maximum particle size of $60 \mu \mathrm{m}$. The CNTs dispersion through matrix was made using two approaches: (1) the dispersion of CNTs and mixture with Al powders performed in a single step as describe in previous work [3] and (2) the CNTs were untangle previously by ultrasonication in propanol for 15 min and then mixed with Al powders by ball milling in propanol during $6 \mathrm{~h}$ with a ball-to-powder ratio of 20:1. Microstructural characterization of Al-CNTs nanocomposites was performed by optical microscopy (OM), scanning and transmission electron microscopy (SEM and TEM), electron backscattered diffraction (EBSD) and high resolution transmission electron microscopy (HRTEM). To evaluate the CNTs dispersion, an area of $1609182 \mu^{2}$ was analysed for each sample using an optical microscope and the software Leica Application Suite.

Microstructural characterization by SEM of the nanocomposites reveals that the CNTs are mainly observed in small clusters at grain boundary junctions. As can be seen in the SEM images of Figures 1a and 1b, samples with CNTs dispersed by ball milling technique apparently exhibit smaller CNT clusters. The analysis of OM images reveals that the average size of the CNT agglomerates is $5.1 \mu \mathrm{m}$ for the both samples. The samples produced with CNTs dispersed by ultrasonication and ball-milling have agglomerates with a maximum size of 76 and $69 \mu \mathrm{m}$, respectively. These results confirm the ones observed in the SEM images. Based on these, it is can be concluded that with the use of ball milling for mixing CNTs with Al powders can improve the CNTs dispersion by reducing the size of the agglomerates. The nanocomposites were also observed through TEM and HRTEM. It is essential to make these observations in order to analyze the distribution of CNTs through the matrix and identify any nanometric phase present. TEM and HRTEM images revealed some of CNTs embedded into the matrix, inside the aluminum grains. The formation of $\mathrm{Al}_{4} \mathrm{C}_{3}$ phase due to the reaction between the CNTs and the Al, was also identified by HRTEM observations. The Inverse Pole Figure maps of Al-CNTs nanocomposites produced with CNTs mixing by ball milling can be seen in Figure 2; the color of the grains indicates their crystallographic orientation. In this Figure, the lack of predominance of one color, or colors, indicates that the Al-CNTs nanocomposites do not have a preferred crystallographic orientation (texture); i.e. the incorporation of CNTs does not promote the formation of a texture. These results are similar to the results observed in previous studies [3] for the nanocomposites produced with CNTs dispersed with ultrasonication [4]. 


\section{References:}

[1] K.U. Kainer, in "Metal Matrix Composites: Custom-made Materials for Automotive and Aerospace Engineering”, (WILEY-VGH Verlag GmbH \& Co. KGaA. Weinheim) 2006.

[2] Nuno Silvestre, International Journal of Composite Materials 3 (2013) p. 28.

[3] S. Simões, F. Viana, M. A. L. Reis and M. F. Vieira, Composites Structures 108 (2014) p. 992.

[4] This research is sponsored by FEDER funds through the program COMPETE - Programa Operacional Factores de Competitividade - and by national funds through FCT - Fundação para a Ciência e a Tecnologia -, under the project PEst-C/EME/UI0285/2013 and the project PTDC/EMETME/122472/2010.

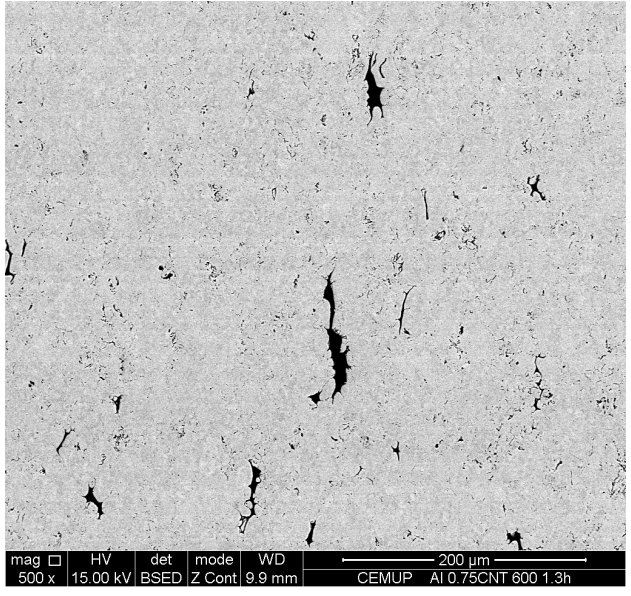

(a)

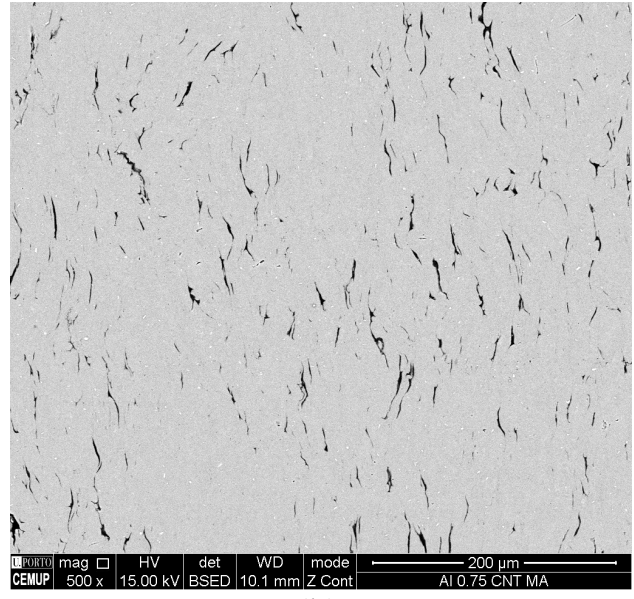

(b)

Figure 1. SEM images of Al-CNTs nanocomposites produced with (a) CNTs dispersed and mixing with Al powders by ultrasonication (b) CNTs mixing with Al powders by ball milling.

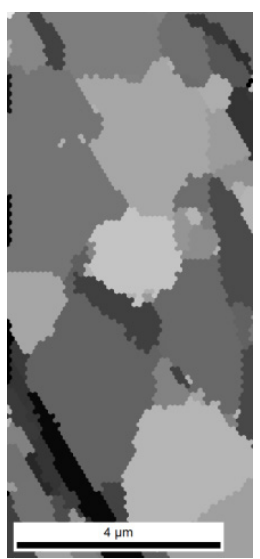

(a)

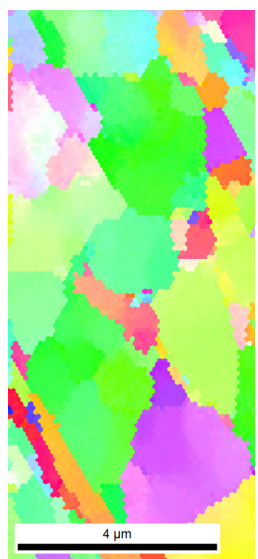

(b)

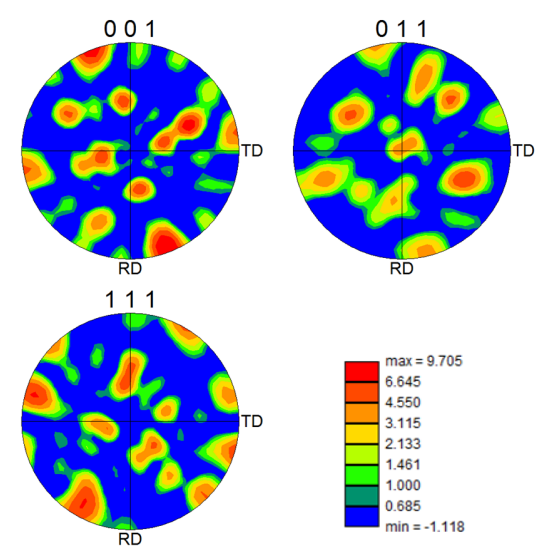

(c)

Figure 2. EBSD results for nanocomposites produced with CNTs mixing with Al powders by ball milling (a) grains image map, (b) inverse pole figure map and (c) pole figures. 